\title{
Advanced proteomics procedure as a detection tool for predictive screening in type 2 pre-Diabetes
}

\author{
Jadranka Koehn • Kurt Krapfenbauer
}

Received: 2 December 2009 / Accepted: 28 January 2010 / Published online: 24 March 2010

(C) European Association for Predictive, Preventive and Personalised Medicine 2010

\begin{abstract}
It has been suggested that a more precise selection of predictive biomarkers may prove useful in the early diagnosis of type 2 diabetes (T2D), even when glucose tolerance is normal. This is vital since many T2D cases may be preventable by avoiding those factors that trigger the disease process (primary prevention) or by use of therapy that modulates the disease process before the onset of clinical symptoms (secondary prevention) occurs. The selection of predictive markers must be carefully assessed and depends mainly on three important parameters: sensitivity, specificity and positive predictive value. Unfortunately, biomarkers with ideal specificity and sensitivity are difficult to find. One potential solution is to use the combinatorial power of different biomarkers, each of which alone may not offer satisfactory specificity and sensitivity. Recent technological advances in proteomics and bioinformatics offer a great opportunity for the discovery of different potential predictive markers. In this review, we described a cellular T2D model as an example with the intent of providing specific enrichment and new identification strategies, which might have the potential to improve predictive biomarker identification and to bring accuracy in disease diagnosis and classification, as well as therapeutic monitoring in the early phase of T2D.
\end{abstract}

Keywords Diabetes mellitus · Beta-cell failure . Glycotoxicity · Lipotoxicity · Predictive biomarker . Pancreatic polypeptide Y. Proteomics

\footnotetext{
J. Koehn $\cdot$ K. Krapfenbauer $(\square)$

Department of Cranio-Maxillofacial and Oral Surgery,

Medical University of Vienna,

Waehringer Guertel 18-20,

1090 Vienna, Austria

e-mail: kurt.krapfenbauer@boehringer-ingelheim.com
}

\section{General introduction}

Type 2 diabetes (T2D) is one of the fastest growing health concerns worldwide with a prevalence of approximately $5 \%$ in the United States (American Diabetes Association, 2002). Due to sedentary lifestyle, unhealthy nutrition and a prolonged lifespan, the prevalence in the developed and developing countries is still increasing. Health experts have warned of a global epidemic of diabetes caused by a rise in overweight and obesity. There are currently $120-140$ million people worldwide with T2D, and if trends continue, this number is predicted to double in the next 25 years. In the Western world, around $90 \%$ of T2D cases are attributable to weight gain. Because of the severe health and cost implications of this disease, organizations such as the International Diabetes Federation (IDF) have called for increased efforts to prevent its development. The IDF estimates that 314 million people worldwide, or $8.2 \%$ of the global population, have impaired glucose tolerance (IGT), a state that often precedes T2D. In addition, T2D is a heavily under-diagnosed disease and is usually only discovered at a late stage, when the first complications occur. Detection in an early state would be highly desirable, as the progression of T2D can then be slowed down by simple, nonpharmacologic means, i.e. weight loss and exercise. The existing diagnostic tests, fasting plasma glucose (FPG), the oral glucose tolerance (OGT) test or the euglycemic hyperinsulinemic clamp are either relatively insensitive and inaccurate or so difficult to perform that they do not lend themselves to mass screening.

The identification of a reliable, accurate, cost-effective and non-invasive test for T2D that could be applied in the prediabetic state would present a sizeable opportunity for diagnostics and would be valuable as a monitoring marker for clinical studies in T2D. Although significant progress 
has been made in the treatment of T2D, new insulin sensitizing drugs such as the thiazolidinediones display significant side-effects and major health concerns still prevail for this class of compounds. Most importantly, there is no medication on the market that slows down the progressive beta-cell ( $\beta$-cell) failure, a hallmark of latestage T2D. The identification of pathways and factors involved in either insulin-resistance of skeletal muscle or $\beta$ cell failure could lead to new drug targets and the development of novel pharmaceuticals for the treatment of impaired glucose tolerance (IGT) and for the prevention of $\beta$-cell failure, respectively.

Beta-cell failure is explained by both a relative loss of $\beta$ cell mass as well as secretory defects including enhanced basal insulin secretion by the $\beta$-cells and a selective loss of sensitivity to insulin mainly in skeletal muscle but also in other organs. Beta-cell failure is the last step in the progression of type 2 diabetics towards dependence on external insulin. The loss of $\beta$-cell function is believed to be triggered by long-term exposure to enhanced levels of glucose and lipids (glycol- and lipotoxicity). However, the molecular mechanisms of this phenomenon are poorly understood. Although insulin sensitizers hold some promise, there is currently no proven treatment for T2D patients that would prevent progression towards $\beta$-cell failure. It would also be useful to identify better targets and/or predictive markers for $\beta$-cell failure or function that are more sensitive or more reliable than the targets/markers commonly used, such as proinsulin or its processing products insulin or C-peptide. This is vital since many T2D cases may be preventable by avoiding those factors that trigger the disease process (primary prevention) or by use of therapy that modulates the disease process before the onset of clinical symptoms (secondary prevention) occurs. For a better understanding of the molecular events leading to the loss of $\beta$-cell function and to define possible targets for a function-preserving treatment a cellular model for $\beta$ cell failure is thus required. Furthermore, there is currently no good test available to assess $\beta$-cell functionality. Such a test would be of great value in potential clinical trials for pharmaceuticals that preserve $\beta$-cell function. For this reason, several labs systematically analyzed $\beta$-cell failure to identify significant changes that occur after exposure to both palmitate and high glucose [1].

The rat insulinoma cell line INS-1 displays $\beta$-cell-like features making it a good in vitro model for this cell type. It has preserved many $\beta$-cell characteristics, including glucose-stimulated insulin secretion. Furthermore, study of these $\beta$-cells with decreased insulin secretion as compared to cells with normal function could lead to a better understanding of the biology of $\beta$-cell failure and could highlight possible targets for therapeutic intervention. Study of these cells may also lead to the identification of markers for $\beta$ - cell failure or function that are more reliable or sensitive than the C-peptide.

In conclusion, important steps were made towards the identification of $\beta$-cell specific proteins that might serve in the future as predictive markers for $\beta$-cell function or failure. Furthermore, it would be an advantage to identify such markers that can be detected in plasma of humans. Follow-up experiments using animal models and human blood and plasma samples were designed to validate opportunities to pharmaceutically target $\beta$-cell failure and to generate novel medicines for T2D.

\section{Current status of diabetes diagnostics}

Blood glucose testing is the most commonly used method for the diagnosis and monitoring of diabetes mellitus. FPG levels and plasma glucose levels $2 \mathrm{~h}$ after the uptake of a $75 \mathrm{~g}$ dose of glucose are two possible methods to detect the disease. Two independently obtained positive test results are required to establish the diagnosis. As it is easier and less time-consuming to perform, the FPG test is recommended by the American Diabetes Association. However, FPG levels will only be increased, when an increased insulin secretion fails to compensate for insulin resistance, which will only be the case in late stages of T2D. The OGT test and the euglycemic hyperinsulinemic clamp [1] are two methodologies that have the potential to detect insulin resistance at an earlier stage, but are relatively time-consuming to perform and are not amenable to mass testing. Once the diagnosis is established, the efficacy of a treatment regimen can be monitored by measuring glycated hemoglobin (HbAlc) which reflects the plasma glucose levels over the last 4 6 weeks [2]. For an assessment of $\beta$-cell function, the measurement of the concentration of C-peptide is, at this time, the best method available [3, 4]. Measurement of this parameter is however not recommended for clinical practice as it suffers from a large variability [5].

\section{Current biomarkers for diabetes}

The classical risk factors for type 1 diabetes (T1D) are, apart from familiar predisposition, genetic markers, like certain HLA-DQ or CTLA4 genotypes associate with increased risk of developing T1D. The detection of auto antibodies to islet cell antigens (ICA), insulin, glutamic acid decarboxylase (GAD-65), or IA-2 protein tyrosine phosphatase marks the onset of the autoimmune disease and can be detected years before clinical symptoms of diabetes appear. While certain variants in genes encoding a $\beta$-cell specific ion-channel (SUR8/Kir6.2) are associated with transient or permanent neonatal diabetes and mutations in the ABCA1 gene have recently been associated with $\mathrm{T} 2 \mathrm{D}$ (reviewed in [6]), obesity is still the greatest known risk 
factor for T2D. Though familiar clustering of T2D implies the existence of genetic risk factors, these are still unknown.

While for T1D early diagnostic markers exist in the form of auto antibodies, T2D can so far only be diagnosed when the clinical symptoms appear at the later stages of the disease. Metabolic markers like hyperglycemia, insulin or C-peptide level, and HbAlc are used to diagnose diabetes and monitor the disease state in all types of diabetes. Since an early diagnosis and an early therapy hold the greatest promises to postpone full disease development and to delay secondary morbidities like cardiovascular diseases, reliable, sensitive markers for early T2D are a key concern.

Several novel biomarkers for T2D have been reported and reviewed recently [7]. Among the T2D marker candidates are markers of fatty liver routinely tested like alanine aminotransferase (ALT) and $\gamma$-glutamyl transferase $(\gamma-\mathrm{GT})$, both well correlated with diabetes risk [8]. Low levels of sex homone binding globuline (SHBG), a hepatically secreted hormone negatively regulated by insulin, can indicate high insulin levels, insulin resistance and risk of $\mathrm{T} 2 \mathrm{D}$, a correlation that is stronger in women than in men [9]. Low SHBG has been reported to be predictive of gestational diabetes [10]. Inflammatory markers interleukin 6 (IL-6) and C-reactive protein (CRP) induced by IL-6 have also been associated with T2D [11], as has another acute phase marker ferritin [12]. Some studies suggested that CRP and IL-6 levels positively correlated with insulin resistance and with the risk of acquiring type 2 DM. However there is no correlation between plasma ferritin level and glycemic control or diabetic microangiopathic complications. Serum ferritin, initially a marker of cellular iron stores, is therefore not fully validated as a indicator of T2D risk [13]. Adiponectin, a hormone produced by adipocytes and involved in the regulation of fat and glucose metabolism, has an inverse correlation to diabetes, i.e. low adiponectin levels correlate with high T2D risk [14] and genetic variants of adiponectin have been associated with increased risk of T2D [15]. Adiponectin exerts insulinsensitizing and anti-infamatory functions through two different seven-transmembrane receptors expressed predominantly in $\beta$-cells and skeletal muscles (ADIPOR1) or the liver (ADIPOR2) [16]. Leptin, another adipocyte hormone regulates appetite and has been associated with T2D but the significance is unclear, as leptin levels can be deregulated in leptin resistant obese patients and a positive and a negative association with T2D can be found [17]. Tissue plasminogen activator (tPA) and its inhibitor PAI-1 have also been linked to T2D [18-20]. Both of these proteins can be secreted form endothelial cells and high plasma levels usually reflect endothelial stress or inflammation. PAI-1 can also be released from adipocytes and hepatocytes and its plasma levels increase with adiposity, weakening the association with T2D. Like tPA and PAI-1 von Willebrand factor (vWF) is a maker of endothelial dysfunction associated with risk of T2D in the literature [21] and like the former it is induced by proinflammatory cytokines. The association of vWF with T2D is less robust and significance is lost after adjustment for obesity or inflammatory cytokines in some studies [20]. Other risk factors for T2D have been described, many of these are also associated with obesity or inflammation and thus might not be useful to discern an obese patient at risk to develop T2D form one not at risk. Furthermore, many risk factors have been analyzed only in small study groups. All risk factors identified so far are present in healthy populations as well though at a different levels. But it is not only T2D that will influence their expression level, age, sex, ethnicity might also play a role increasing the variability and reducing the specificity.

\section{Current treatment scheme for T2D}

In the early stages of the disease, weight loss and an increase in physical activity are often sufficient to restore insulin sensitivity (American Diabetes Association, 2002). If this regimen fails, non-insulinotropic agents such as acarbose or metformin (and glitazones) are the first-line therapeutics. When insulin levels drop, insulin secretagogues (sulfonylureas, repaglinide) need to be added to the therapeutic regimen. Eventually, when this regimen has become insufficient, insulin injection becomes necessary.

Scientific rationale for $\beta$-cell failure as a disease model for $\mathrm{T} 2 \mathrm{D}$

Beta-cell failure occurs at a late stage in the progression of T2D and leads to overt diabetes. The mechanisms by which $\beta$-cell function is impaired are largely unknown but have been shown to involve hyperglycemia as well as hyperlipidemia [22].

Interestingly, $\beta$-cell failure does not appear to be an inevitable consequence of hyperinsulinemia. Some animal models of hyperinsulinemia/diabetes show persistent lifelong hyperinsulinemia (e.g. ob/ob mice, fa/fa rats) while others show deteriorating $\beta$-cell function and apoptosis of $\beta$-cells (e.g. db/db mice, Psammomys obesus, and rhesus monkeys) [23]. The reasons for these differences are unclear and include genetic factors [24]. The situation in humans may be similar, as insulin resistance is an almost inevitable consequence of obesity but only some individuals have frank diabetes and progress into insulin dependence. The fact that $\beta$-cell failure is avoidable in some animal models encourages research towards the prevention of $\beta$-cell failure. In vitro studies have demonstrated a glucotoxic effect that was only partially reversible $[23,25,26]$. 
Lipotoxicity plays an equally important role [27, 28]. A profound effect on $\beta$-cell function is observed in models combining both factors [22]. Some scientists speculate that these two mechanisms are independent of each other and involve distinct pathways (Dr. M. Donath, Zurich, personal communication). Apoptosis plays a critical role in $\beta$-cell failure and T2D [29] and is induced by Fas-FasL interaction where the latter is constitutively expressed by $\beta$-cells and the former induced by cytokines and glucose (Dr. M. Donath, personal communication). FLIP blocks the cascade from Fas-FasL interaction $\rightarrow$ caspase $8 \rightarrow 3$ and finally apoptosis and even reverses the effect of high glucose, thus resulting in proliferation instead of apoptosis. This suggests that prevention of $\beta$-cell failure is feasible. The apoptotic effect of lipids, mediated by Bcl-2 and ceramide, is less well documented. Importantly, although apoptotic pathways have been explored extensively, the present review addresses functional $\beta$-cell failure outside apoptosis. A decrease in $\beta$-cell mass was observed in patients with advanced T2D but not in the early stages of the disease [30]. The metabolic disturbances of most patients will go unnoticed until frank hyperglycemia is detected. However, the vast majority of patients will remain in a stage of partial compensation before moving on to dependence on exogenous insulin. At this particular stage, compounds preventing $\beta$-cell failure could be applied, therefore the goals of our proteomics study are twofold: (i) The first line of experiments will be directed towards the identification and validation of a potential monitoring marker for clinical studies. Indeed, the current gold standard for the measurement of $\beta$-cell function, the measurement of the insulin C-peptide, is not sufficiently reliable for this purpose. To ensure its broad acceptance, a marker would have to be established well in advance of a clinical program aimed at demonstrating disease modifying (i.e., $\beta$-cell failure preventing) efficacy of a novel compound. (ii) Secondly, the changes in protein expression patterns should be studied in order to improve our understanding of the biological processes involved in $\beta$-cell failure, ultimately leading to the identification of pathways and novel targets for therapeutic intervention.

\section{Methodology}

Cellular model system for the identification of potential markers of $\beta$-cell failure

Isolated islets and insulin secreting cell lines become desensitized upon exposure to high levels of glucose and palmitate [22]. The rat insulinoma cell line INS-1 and the mouse cell line Min6 appear to be the best available model cell lines at this time $[31,32]$. They have retained many characteristics of primary $\beta$-cells, including the susceptibility to the combined toxic effect of glucose and lipids.

Two classes of proteins could fulfill the criteria for a marker of $\beta$-cell failure: (i) Highly expressed $\beta$-cell specific proteins could become detectable in the bloodstream upon cell death, and (ii) the level of $\beta$-cell specific secreted proteins could significantly drop as a sign of impaired $\beta$-cell function. Ideally the latter would be constitutively secreted and not depend on glucose concentration as shown in the results of the OGTT. For this purpose, a catalogue of proteins expressed in $\beta$-cell lines was established to identify $\beta$-cell specific proteins that have the potential to become detectable in human blood and that could serve as a marker for $\beta$-cell failure. Beta-cell specific proteins identified by this strategy will have to be first validated in an animal model for $\beta$-cell failure, for example in blood samples collected from rat strains rapidly developing full-blown diabetes (e.g. ZDF rats), before human blood samples can be tested.

New enrichment and identification strategies of differentially regulated proteins in failing $\beta$-cells

Beta-cell failure can be mimicked in several cell culture models by adding a combination of fatty acids and increased concentrations of glucose to the culture medium as shown in $[22,28]$. Comparison of expression patterns from untreated cells, cells treated with either a fatty acid or high glucose, and cells treated with both fatty acid and high glucose should highlight gene products whose regulation correlates with $\beta$-cell failure in a cell culture model. The results from this study directed the proteome comparison towards the compartments that appeared most affected based on the transcript analysis. In a second step these changes can be validated using animal models such as ZDF rats or $\mathrm{db} / \mathrm{db}$ mice. Finally, findings from the primary $\beta$ cells must be further validated by using human plasma samples from different donor groups (e.g. patients with impaired glucose tolerance (IGT), impaired fasting glucose (IFG), type 2 diabetes (T2D), type 1 diabetes (T1D), and healthy donors).

Critical points of the $\beta$-cell failure model:

- A reliable, reproducible model of $\beta$-cell failure induced by gluco- and lipotoxicity after a reasonable exposure time must be established first.

- Triggering failure of the cultured cells to secrete insulin is a time-dependent process that must be carefully monitored (see Fig. 2). Establishing the appropriate monitoring readouts is therefore crucial to this project. Selecting the appropriate time point based on the measurement of the selected parameters is difficult and requires kinetic measurements. 


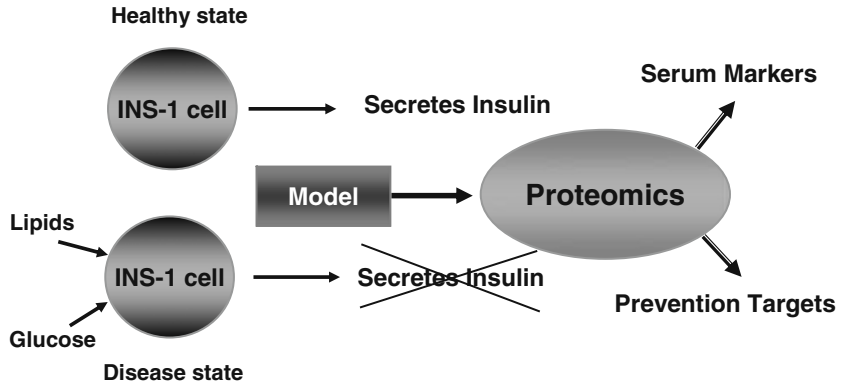

Fig. 1 In vitro model of $\beta$-cell failure: Concept of gluco/lipotoxicity

- Up-scaling of the cell culture is necessary for obtaining sufficient quantities of starting material for a proteome analysis (see Fig. 4). This may lead to variations. Careful monitoring by measurement of the parameters defined by the pilot experiments is therefore mandatory.

- Beta-cell specific proteins, even if highly abundant intracellularly, or secreted proteins may not be present in blood in detectable quantities. Therefore highly sensitive ELISA must be used to validate findings in human blood samples

- A fractionation scheme that efficiently enriches for secretory vesicles has to be established.

- The concentration of secreted proteins in the culture supernatant is likely to be very low. The accessibility of these proteins by mass spectrometric methods should be therefore assessed.

Methodology approach and cellular study design

Features of $\beta$-cell failure can be performed by chronic exposure of $\beta$-cells to a combination of high glucose/fatty acids (FAs), suggesting that hyperlipidemia as well as hyperglycemia may contribute to decompensation of $\beta$-cells. INS- 1 cells pretreated for $30 \mathrm{~h}$ with a combination of $10 \mathrm{mM}$ glucose and $0.5 \mathrm{mM}$ palmitate can be used for these experiments. Establishment of $\beta$-cell culture conditions for proteomics analysis should be carried out in parallel (see Figs. 1 and 3). Screen INS-1 cell cultures under treatment with glucose/fatty acids and identification of different expressed protein levels between glyco/ lipotoxicity and combination. Focus on secreted proteins in the supernatant as possible markers for $\beta$-cell failure/function. Enrichment and identification of secreted INS-1 peptides/ proteins in the medium by heparin chromatography followed by LC-MS were applied.

General introduction and strategies for enrichment and purification of secreted proteins

In order to identify proteins secreted by INS-1 $[31,33]$ two methods can be applied: (i) Fractionation of the cells by differential sedimentation into subcellular compartments with subsequent identification of the proteins based on their peptide mass fingerprint using MALDI-TOF mass spectrometry, and (ii) enrichment of glycoproteins by heparin chromatography followed by one-dimensional SDS-PAGE and identification of proteins by analysis of the tryptic peptides resulting from protein digest by liquid chromatography coupled to tandem mass spectrometry resulting in identification based on protein sequence tags (see Fig. 4). The combination of these two purification strategies allowed us to increase the efficiency of protein identification in the cellular compartments as well as in the medium of cultured cells.

Enrichment of putative secreted proteins by heparin columns from the medium and identification by LC-MS

Visualization and identification of secreted proteins may facilitate the identification of novel drug targets and different biomarkers including predictive markers. However, not all proteins in organism are expressed in amounts sufficient for

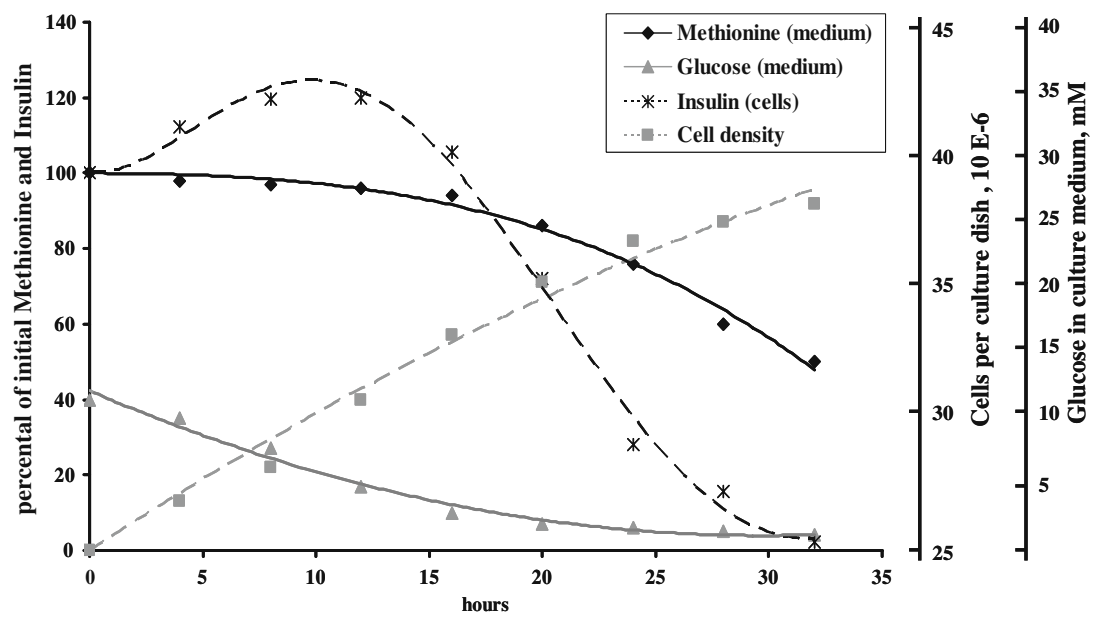

Fig. 2 Characterization and whole protein profiling of INS-1 cells: Model of $\beta$-cell failure induced by gluco- and lipotoxicity after 35 h exposure time 


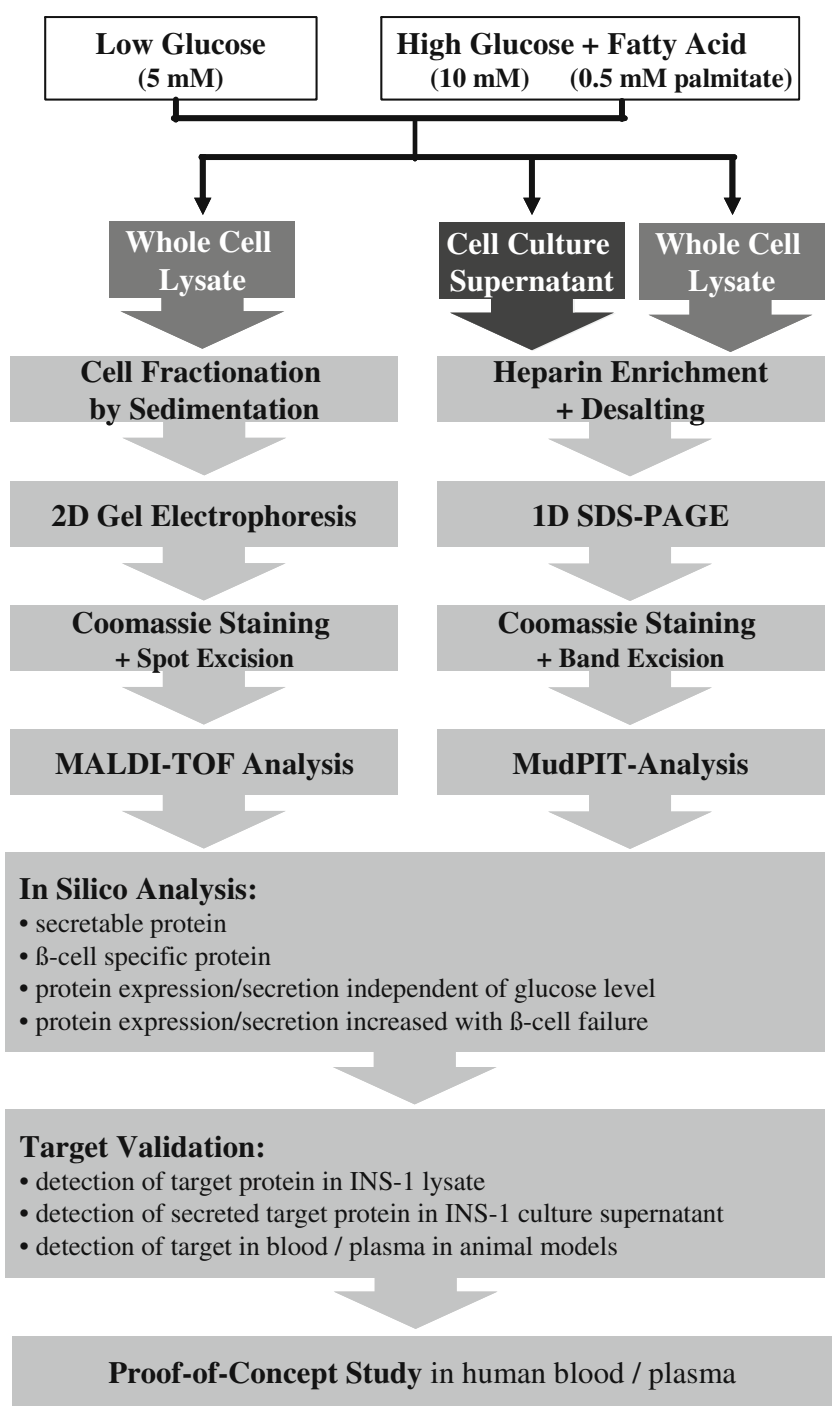

Fig. 3 Flowchart of proteomics approach to identify markers of $\beta$-cell failure using mass spectrometry (MudPIT $=$ Multi protein identification technology and MALDI=matrix assisted laser desorption/ionisation mass spectrometry) in combination with in silico analysis

detection by two-dimensional or one dimensional polyacrylamide gel electrophoresis. In order to visualize and identify low-copy gene products with signaling function, enrichment of low-abundance cytosolic proteins by applying heparin chromatography were chosen because heparin has a high protein binding capacity and can discriminate and enrich proteins with minor differences in their pI-values and glycosylation patterns $[34,35]$. As most secreted proteins are glycosylated, [36] heparin sepharose and/or lectin columns are versatile tools for the enrichment of many classes of glycosylated proteins such as proteins with signaling functions, growth factors, coagulation factors and steroid receptors [37, 38]. The ligand in a heparin sepharose column is a naturally occurring sulfated glycosaminoglycan, which is extracted from the native proteoglycan of porcine intestinal mucosa. Heparin consists of alternating units of uronic acid and D-glucosamine, most of which are substituted with one or two sulphate groups. Immobilized heparin has two main modes of interaction with proteins: It can operate as an affinity ligand; e.g., in its interaction with coagulation factors and it can also function as a high capacity cation exchanger because of its anionic sulphate groups, leading thus to an additional enrichment of positively charged proteins.

To enrich for secreted rat insulinoma proteins with signaling function heparin chromatography was applied prior to proteomic analysis. The column was operated with a syringe instead of a liquid chromatography pump. Elution was performed by increasing the ionic strength with $2 \mathrm{M}$ $\mathrm{NaCl}$. Separation of the eluted proteins was carried out by one-dimensional (1D-) polyacrylamide gel electrophoresis (PAGE) and the proteins were identified by multidimensional protein identification technology (MudPIT) tandem mass spectrometry $[39,40]$ in combination with in silico analysis (see Figs. 4 and 5).

Moreover, heparin chromatography may be useful in the depletion of albumin from body fluids, such as plasma and cerebrospinal fluid, in which it represents more than $50 \%$ of total proteins $[34,41]$. For example, serum albumin, which is represented by a strong band in the starting material (see Fig. 6), was completely recovered in the flow-through fraction. Specific removal of albumin, as well as of other high abundance proteins allowed the visualization and identification of minor components of the samples, whose levels may change in certain disorders [42]. In addition to the easier design of protein purification steps, use of selected chromatography steps prior to the LC-MS/MS, can significantly facilitate the analysis of complex protein mixtures. Methodological sample preparation and purification, as well as systematically identification by MudPIT and MALDI are published in detail $[43,44]$.

Identification of putative secreted proteins by in silico analysis

Secreted proteins are characterized by a signal peptide sequence that helps in threading the protein's $\mathrm{N}$ terminus through a membrane before it is cleaved off by a signal peptidase. Signal peptides are only loosely defined, often with a positively charged polar section, followed by a hydrophobic stretch, and a short pattern around the cleavage site.

The web tool that are used in order to identified or predicted signal peptide from SwissProt, was an developed software tool based on a set of specialized, manually curated Hidden Markov Models (HMMs) that attempt to recognize the (sparse) sequence features common to signal peptides or anchors, respectively [43]. As these sequence signals cannot be reliably predicted, the "signal" and "anchor" scores that any input sequence is assigned are 


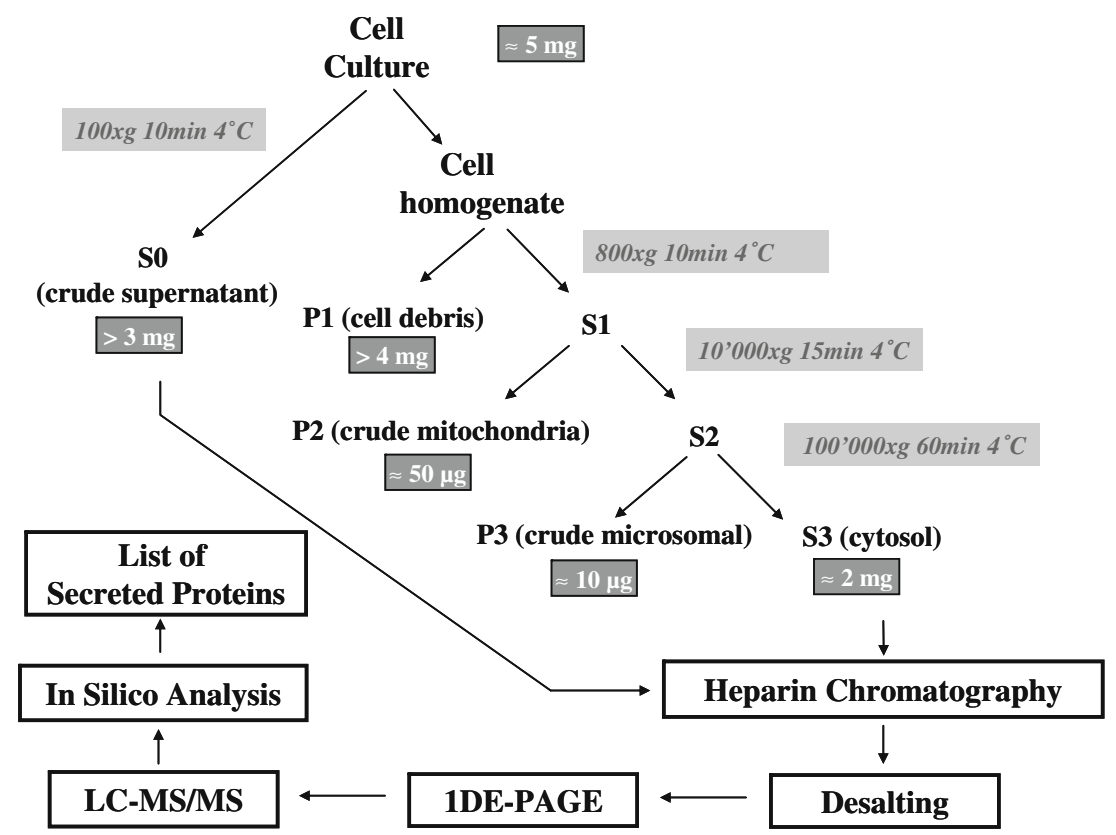

Fig. 4 Scheme of centrifugal prefractionation of rat INS-1 proteins. Different centrifugal force leads to enrichment of cellular components such as mitochondria, microsomal and cytosolic proteins. The

fed into a Support Vector Machine (SVM) in a second analysis step. The SVM was trained on a set of bona fide examples for both classes. On this training set, the SVM obtained the following results on three training sets (signal-anchor-neither):

signal $96 \%$ sensitivity and $96 \%$ specificity anchor $87 \%$ sensitivity and $98 \%$ specificity neither $96 \%$ sensitivity and $97 \%$ specificity cytosolic fraction was subjected to further fractionation by heparin chromatography followed by separation on a $10 \%$ homogenous polyacrylamide gel and protein identification by LC-MS/MS

These results are as good as the best claims in the literature (note, however, that most tools only look at the signal peptides and do not attempt to predict membrane anchors).

For the default setting of this web interface, a list is produced that simply classifies each input sequence in either of the three categories "Signal", "Anchor", or "Normal" (the latter indicating that neither signal peptide nor membrane anchor are predicted).
Fig. 5 2D-PAGE images of differential protein expression patterns in INS- 1 cells incubated under lipo/glucotoxic conditions (10 $\mathrm{mM}$ glucose and 0.5 palimate) versus control $(5 \mathrm{mM}$ glucose). The selected spot of protein (P06303) was identified by MALDI-TOF as pancreatic polypeptide Y [43]
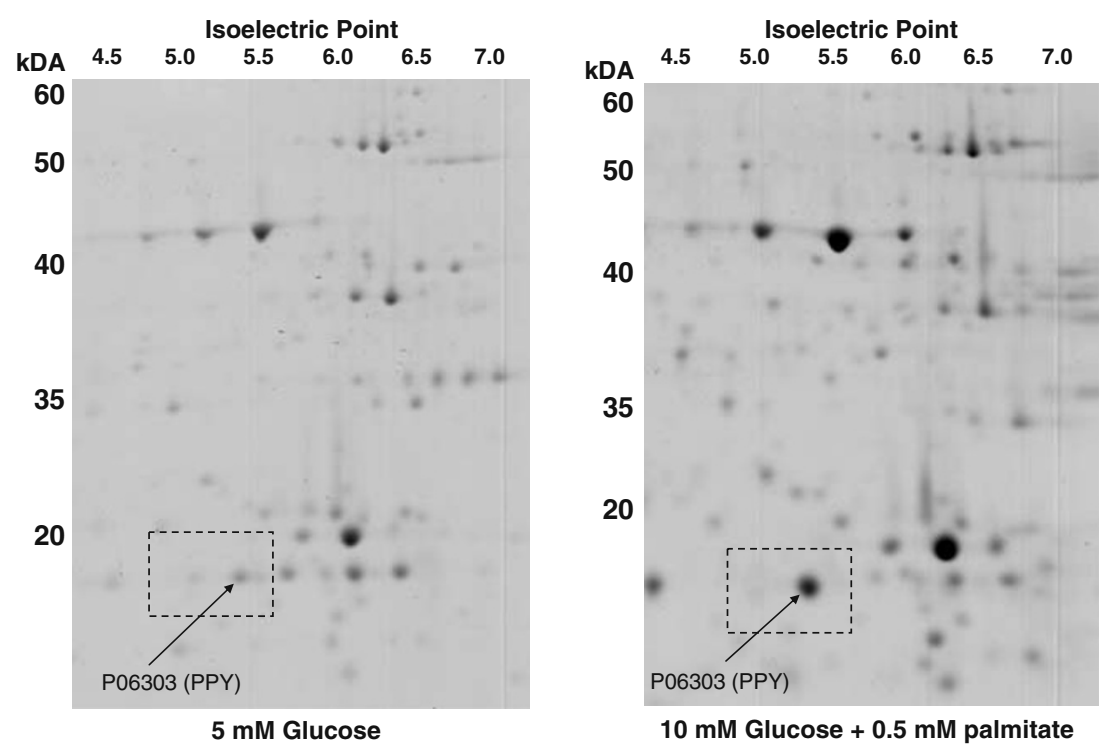


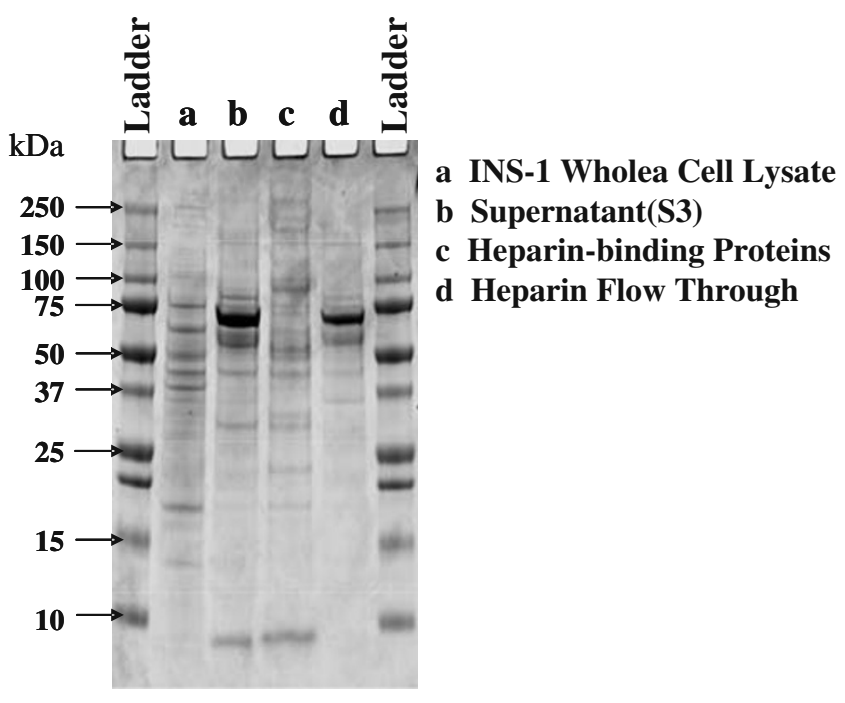

Fig. 6 1DE gel analysis of fractions eluted from heparin chromatography. Fraction enriched with cytosolic rat INS-1 proteins was prepared as stated in [43]. Gels were stained with colloidal Coomassie blue and protein bands were identified by MudPIT [43]

For the "verbose" mode, there is a much more detailed table that has, for every input sequence, the following columns:

seqID, Classification - these are the same as in the default output discussed above.

Confidence - this qualifier can take the values "Unique", "Ambiguous", or "No Class", depending on the SVM results. The SVM determines how well the sequence scores fit its model of either of the three classes. In the "Unique" case, the scores match only one of the three classes, to which the sequence is then assigned. In the "Ambiguous" case, the sequence scores match more than one class-the sequence is assigned to that class into which it fits best. In the "No class" case, the scores do not match the expectations for any of the three classes - the sequence is assigned to that class for which the discrepancy is smallest.

D (Anchor), D (Normal), D (Signal) - these numbers specify the "distances" to a line separating the three classes (to use an intuitive metaphor). For a "Unique" classification, only one of these "distances" is positive (indicating that the sequence is "on the right side of the fence"), whereas the other two are negative ("on the wrong side of the fence" for the two other classes). The larger the numbers, the clearer the classification. For an "Ambiguous" classification, more than one of the D's is positive, for a "No class" classification, all three D's are negative.

SW-classification, SW-note - finally, sequences from the SwissProt database already come with an annotation regarding signal peptides and membrane anchors. For them, we show the SwissProt classifications and any additional comments on them for reference and comparison. Sequences from the HUMANGP, MOUSEGP, and RATGP databases have "none" for both fields.

The HUMANGP, MOUSEGP, and RATGP databases have been completely annotated with this prediction algorithm, the results are displayed in the XR SIGNAL/ ANCHOR annotation lines and listed in Table 1.

\section{Visualization and identification of putative secreted proteins by MudPIT analysis enriched by heparin chromatography}

Heparin binding fractions of cell culture supernatants were analyzed by LC-MS and MALDI-MS and the identification of the putative markers were reported [43]. Five secreted proteins were further confirmed: Chromogranin b, Pancreatic Polypeptide (PPY), Phospholipase A2, Fibronectin and Vinculin were clearly shown to be secreted by INS-1. The amount of secretion into the medium correlated strongly to the insulin content of the INS-1 $\beta$-cells. Based on the organ specify, PPY seemed to be one of the most interesting candidates and was selected for further validation by ELISA. PPY is a secretory glycoprotein with a widespread distribution mostly expressed in endocrine pancreas. These data were underlined by additional OGTT studies which corroborate the findings and show clearly, that PPY is secreted indepented from the glucose level in blood (see Fig. 8). Furthermore it has been suggested that PPY has an autocrine inhibitory effect on iAPP and insulin secretion by different intra- and extracellular mechanism [45]. Ten proteins were selected for further OGTT evaluation. Evaluation/validation by immunoassays in a well-characterized patient population was performed only with PPY. Pancreatic Polypeptide levels are higher in diabetics than controls. A more profound analysis has to demonstrate that the levels correlate with $\beta$-cell failure and not other diabetes-related phenomena (see Fig. 7). A priority application was made for all candidates before a full validation was done.

\section{Detection of PPY in human plasma-Proof-of-Concept}

Clinical utility of the novel marker PPY was assessed by measuring its levels in 10 diabetic patients depending on injections of exogenous insulin and comparing the levels with those measured in 10 patients with demonstrated normal $\beta$-cell function [43].

The OGTT has fallen into disfavor as a tool to examine the mechanism responsible for impaired glucose metabolism in T2D subjects. The rate of glucose absorption varies considerable from one subject to another and cannot be quantified easily. Consequently, the rise in plasma glucose 
Table 1 List of identified secreted INS-1 proteins enriched by heparin chromatography [43]

\begin{tabular}{|c|c|c|c|c|c|}
\hline Fraction & SW.-Nr. & Access. Nr. & Status & Full Protein Name & $\mathrm{kDa}$ \\
\hline Sup. & S106_RAT & P05964 & Sec. & Calcyclin (Prolactin receptor associated protein). & 10.1 \\
\hline Hep. & KV5C_MOUSE & P01635 & Sec. & Ig kappa chain $\mathrm{V}-\mathrm{V}$ region $\mathrm{K} 2$ precursor (Fragment). & 12.6 \\
\hline Sup. & TL19_MOUSE & Q9CQU0 & Sec. & Thioredoxin-like protein p19 precursor & 19.0 \\
\hline Sup. & CYPC_MOUSE & P30412 & Sec. & Peptidyl-prolyl cis-trans isomerase C (EC 5.2.1.8) & 22.8 \\
\hline Hep. & CYPB_RAT & P24368 & Sec. & Peptidyl-prolyl cis-trans isomerase B prec. (EC 5.2.1.8) & 23.0 \\
\hline Hep. & TIM2_RAT & P30121 & Sec. & Metalloproteinase inhibitor 2 precursor (TIMP-2) & 24.3 \\
\hline Hep.; Sup. & TRY1_RAT & P00762 & Sec. & Trypsin I, anionic precursor (EC 3.4.21.4) & 25.9 \\
\hline Hep.; Sup. & TRY2_MOUSE & P07146 & Sec. & Trypsin II, anionic precursor (EC 3.4.21.4) & 26.2 \\
\hline Hep. & PAHO_RAT & P06303 & Sec. & Pancreatic prohormone prec. (Pancreatic polypeptide) & 27.1 \\
\hline Hep.; Sup. & IBP4_RAT & P21744 & Sec. & Insulin-like growth factor binding protein 4 (IGFBP-4) & 27.7 \\
\hline Hep. & ER29_RAT & P52555; P80749 & Sec. & Endoplasmic reticulum protein ERp29 prec. (ERp31). & 28.6 \\
\hline Sup. & PDX4_MOUSE & O08807; & Sec. & Peroxiredoxin 4 (EC 1.11.1.-) (Prx-IV) & 31.0 \\
\hline Hep.; Sup. & SPRC_RAT & P16975; O08953 & Sec. & SPARC precursor & 34.4 \\
\hline Hep. & FSL1_RAT & Q62632 & Sec. & Follistatin-related protein 1 prec. (Follistatin-like 1). & 34.6 \\
\hline Hep.; Sup. & OSTP_RAT & P08721 & Sec. & Osteopontin precursor (Secreted phosphoprotein 1) & 34.9 \\
\hline Hep.; Sup. & DRNG_MOUSE & O55070 & Sec. & Deoxyribonuclease gamma precursor (EC 3.1.21.-) & 35.7 \\
\hline Sup. & CALU_RAT & $\mathrm{O} 35783$ & Sec. & Calumenin precursor (Crocalbin) & 37.0 \\
\hline Sup. & CATM_MOUSE & Q9JL96; Q91Z75 & Sec. & Cathepsin M precursor (EC 3.4.22.-). & 37.4 \\
\hline Sup. & CATB_RAT & P00787; & Sec. & Cathepsin B precursor (EC 3.4.22.1) (Cathepsin B1) & 37.4 \\
\hline Hep. & CATL_RAT & P07154 & Sec. & Cathepsin L prec. (EC 3.4.22.15) & 37.6 \\
\hline Hep. & IF35_MOUSE & Q9DCH4 & Sec. & Eukaryotic translation initiation factor 3 subunit 5 & 38.0 \\
\hline Sup. & RCN1_MOUSE & Q05186 & Sec. & Reticulocalbin 1 precursor. & 38.1 \\
\hline Sup. & DKK3_MOUSE & Q9QUN9 & Sec. & Dickkopf related protein-3 precursor (Dkk-3) & 38.4 \\
\hline Hep. & PGS2_RAT & Q01129 & Sec. & Decorin precursor (Bone proteoglycan II) & 39.8 \\
\hline Sup. & CB45_RAT & Q91ZS3 & Sec. & $45 \mathrm{kDa}$ calcium-binding protein precursor (Cab45) & 42.0 \\
\hline Hep. & SELP_RAT & P25236 & Sec. & Selenoprotein P precursor (SeP). & 42.6 \\
\hline Hep. & PAI1_RAT & P20961 & Sec. & Plasminogen activator inhibitor-1 precursor (PAI-1) & 45.0 \\
\hline Sup. & T11B_RAT & O08727 & Sec. & Tumor necrosis factor recept. superfamily member11B & 46.2 \\
\hline Hep.; Sup. & PEDF_MOUSE & P97298 & Sec. & Pigment epithelium-derived factor precursor (PEDF) & 46.2 \\
\hline Hep. & HS47_RAT & P29457 & Sec. & $47 \mathrm{kDa}$ heat shock protein prec. & 46.5 \\
\hline Sup. & MFGM_RAT & P70490 & Sec. & Lactadherin precursor (Milk fat globule-EGF factor 8) & 47.4 \\
\hline Hep.; Sup. & CRTC_RAT & P18418; P10452 & Sec. & Calreticulin precursor (CRP55) (Calregulin) (HACBP) & 48.0 \\
\hline Hep. & LGMN_RAT & Q9R0J8 & Sec. & Legumain precursor (EC 3.4.22.34) & 49.4 \\
\hline Sup. & NCB2_RAT & Q9JI85 & Sec. & Nucleobindin 2 precursor (DNA-binding protein NEFA). & 50.1 \\
\hline Hep. & PCO1_RAT & O08628 & Sec. & Procollagen C-proteinase enhancer protein precursor & 50.2 \\
\hline Sup. & VAS1_RAT & O54715 & Sec. & Vacuolar ATP synthase subunit S1 prec. (EC 3.6.3.14) & 51.1 \\
\hline Hep. & HRA1_MOUSE & Q9R118 & Sec. & Serine protease HTRA1 precursor (EC 3.4.21.-). & 51.2 \\
\hline Hep. & CLUS_RAT & P05371 & Sec. & Clusterin precursor (Sulfated glycoprotein 2) (SGP-2) & 51.3 \\
\hline Hep.; Sup. & SRPX_RAT & Q63769 & Sec. & Sushi repeat-containing protein SRPX prec. & 51.5 \\
\hline Hep. & ANT3_MOUSE & P32261 & Sec. & Antithrombin-III precursor (ATIII). & 52.0 \\
\hline Hep. & LIPL_RAT & Q06000 & Sec. & Lipoprotein lipase precursor (EC 3.1.1.34) (LPL). & 53.0 \\
\hline Hep. & SG3_MOUSE & P47867 & Sec. & Secretogranin III precursor (SgIII). & 53.3 \\
\hline Hep. & NCB1_RAT & Q63083; P97623 & Sec. & Nucleobindin 1 precursor (CALNUC) & 53.5 \\
\hline Hep. & ARSA_MOUSE & P50428 & Sec. & Arylsulfatase A precursor (EC 3.1.6.8) (ASA) & 53.7 \\
\hline Hep.; Sup. & PRTP_MOUSE & P16675 & Sec. & Lysosomal protective protein precursor (EC 3.4.16.5) & 53.8 \\
\hline Hep.; Sup. & PLTP_MOUSE & P55065; Q99L70 & Sec. & Phospholipid transfer protein precursor & 54.4 \\
\hline Sup. & DPP2_RAT & Q9EPB1 & Sec. & Dipeptidyl-peptidase II precursor (EC 3.4.14.2) & 55.1 \\
\hline Hep. & CPS1_MOUSE & P03940 & Sec. & Cytochrome P450 XXIA1 (EC 1.14.99.10) & 55.3 \\
\hline Sup. & PDA3_RAT & P11598 & Sec. & Protein disulfide isomerase A3 precursor (EC 5.3.4.1) & 56.6 \\
\hline
\end{tabular}


Table 1 (continued)

\begin{tabular}{|c|c|c|c|c|c|}
\hline Fraction & SW.-Nr. & Access. Nr. & Status & Full Protein Name & $\mathrm{kDa}$ \\
\hline Sup. & PDI_RAT & P04785; P13700 & Sec. & Protein disulfide isomerase precursor (PDI) & 56.9 \\
\hline Hep. & MM19_MOUSE & Q9JHI0 & Sec. & Matrix metalloproteinase-19 precursor (EC 3.4.24.-) & 59.1 \\
\hline Hep.; Sup. & HEXA_MOUSE & P29416; Q64246 & Sec. & Beta-hexosaminidase alpha chain prec. (EC 3.2.1.52) & 60.6 \\
\hline Sup. & TPP1_RAT & Q9EQV6 & Sec. & Tripeptidyl-peptidase I precursor (EC 3.4.14.9) (TPP-I) & 61.3 \\
\hline Hep. & GPC1_RAT & P35053 & Sec. & Glypican-1 precursor (HSPG M12). & 61.7 \\
\hline Sup. & ALBU_RAT & P02770; P11382 & Sec. & Serum albumin precursor & 68.7 \\
\hline Sup. & ASM_MOUSE & Q04519 & Sec. & Sphingomyelin phosphodiesterase prec. (EC 3.1.4.12) & 69.9 \\
\hline Hep. & SPL1_RAT & P24054 & Sec. & SPARC-like protein 1 prec. (Matrix glycoprotein Sc1). & 70.6 \\
\hline Hep.; Sup. & KNG_RAT & P08934; Р08933 & Sec. & Kininogen precursor & 70.9 \\
\hline Sup. & IDUA_MOUSE & P48441 & Sec. & Alpha-L-iduronidase precursor (EC 3.2.1.76). & 71.1 \\
\hline Hep. & GR78_RAT & P06761 & Sec. & 78 kDa glucose-regulated protein precursor (GRP 78) & 72.3 \\
\hline Hep. & PDA4_RAT & P38659 & Sec. & Protein disulfide isomerase A4 precursor (EC 5.3.4.1) & 72.8 \\
\hline Hep.; Sup. & BGLR_MOUSE & P12265; Q61601 & Sec. & Beta-glucuronidase precursor (EC 3.2.1.31). & 74.2 \\
\hline Hep. & FBL1_MOUSE & Q08879; Q08878 & Sec. & Fibulin-1 prec. (Basement-membrane protein 90) & 78.0 \\
\hline Hep. & SM3B_MOUSE & Q62177 & Sec. & Semaphorin 3B precursor (Semaphorin A) & 82.8 \\
\hline Sup. & PLO1_RAT & Q63321 & Sec. & Procollagen-lysine,2-oxoglutarate 5-dioxygenase 1 & 83.6 \\
\hline Hep. & LOL3_MOUSE & Q9Z175; Q9JJ39 & Sec. & Lysyl oxidase homolog 3 precursor (EC 1.4.3.-) & 83.6 \\
\hline Hep.; Sup. & AD10_MOUSE & O35598 & Sec. & ADAM 10 precursor (EC 3.4.24.-) & 83.9 \\
\hline Sup. & MEPA_MOUSE & P28825 & Sec. & Meprin A alpha-subunit precursor (EC 3.4.24.18) & 84.1 \\
\hline Hep. & PLO3_MOUSE & Q9R0E1 & Sec. & Procollagen-lysine,2-oxoglutarate 5-dioxygenase 3 & 84.9 \\
\hline Hep.; Sup. & A4_RAT & P08592 & Sec. & Alzheimer's disease amyloid A4 protein homolog & 86.6 \\
\hline Sup. & APP2_RAT & P15943 & Sec. & Amyloid-like protein 2 precursor & 86.8 \\
\hline Hep. & ENPL_MOUSE & P08113; P11427 & Sec. & Endoplasmin prec. (Endoplasmic reticulum protein 99) & 92.4 \\
\hline Sup. & DAG1_MOUSE & Q62165; Q61094 & Sec. & Dystroglycan precursor & 96.8 \\
\hline Hep. & FPRP_RAT & Q62786 & Sec. & Prostaglandin F2 receptor negative regulator precursor & 98.7 \\
\hline Hep. & NAH5_RAT & Q9Z0X2 & Sec. & Sodium/hydrogen exchanger $5 \mathrm{Na}(+) / \mathrm{H}(+)$ exchanger & 99.0 \\
\hline Hep.; Sup. & DSC3_MOUSE & P55850; O55110 & Sec. & Desmocollin 3 precursor. & 100.6 \\
\hline Hep. & TSP3_MOUSE & Q05895 & Sec. & Thrombospondin 3 precursor. & 103.9 \\
\hline Hep.; Sup. & PEX6_RAT & P54777; O55097 & Sec. & Peroxisome assembly factor-2 (PAF-2) & 104.4 \\
\hline Hep. & ITH2_MOUSE & Q61703 & Sec. & Inter-alpha-trypsin inhibitor heavy chain $\mathrm{H} 2$ precursor & 105.9 \\
\hline Hep. & CA16_MOUSE & Q04857 & Sec. & Collagen alpha $1(\mathrm{VI})$ chain precursor. & 108.4 \\
\hline Hep. & AMD_RAT & P14925; P70710 & Sec. & Peptidyl-glycine alpha-amidating monooxygenase & 108.6 \\
\hline Sup. & CLS1_MOUSE & Q9EPL2 & Sec. & Calsyntenin-1 precursor. & 108.8 \\
\hline Sup. & OXRP_RAT & Q63617 & Sec. & $150 \mathrm{kDa}$ oxygen-regulated protein precursor (Orp150) & 111.2 \\
\hline Hep. & M2B1_MOUSE & O09159; O55037 & Sec. & Lysosomal alpha-mannosidase precursor EC 3.2.1.2 & 114.5 \\
\hline Sup. & M2B2_MOUSE & O54782 & Sec. & Epididymis-specific alpha-mannosidase (EC 3.2.1.24) & 115.6 \\
\hline Hep.; Sup. & TSP1_MOUSE & P35441 & Sec. & Thrombospondin 1 precursor. & 129.6 \\
\hline Hep.; Sup. & LMG2_MOUSE & Q61092 & Sec. & Laminin gamma-2 chain precursor & 130.1 \\
\hline Hep. & DIA1_MOUSE & O08808 & Sec. & Diaphanous protein homolog 1 & 139.3 \\
\hline Sup. & RGSC_RAT & O08774; O88383 & Sec. & Regulator of G-protein signaling 12 (RGS12). & 150.4 \\
\hline Hep.; Sup. & A2MG_RAT & P06238 & Sec. & Alpha-2-macroglobulin precursor (Alpha-2-M). & 163.6 \\
\hline Sup. & A1I3_RAT & P14046 & Sec. & Alpha-1-inhibitor III precursor. & 163.7 \\
\hline Hep.; Sup. & LMG3_MOUSE & Q9R0B6 & Sec. & Laminin gamma-3 chain precursor & 172.2 \\
\hline Hep. & LMG1_MOUSE & P02468 & Sec. & Laminin gamma-1 chain precursor (Laminin B2 chain). & 177.2 \\
\hline Sup. & CO3_RAT & P01026 & Sec. & Complement $\mathrm{C} 3$ precursor & 186.3 \\
\hline Sup. & LTB1_RAT & Q00918 & Sec. & Latent transforming growth factor beta binding prot. 1 & 186.5 \\
\hline Hep. & LMB1_MOUSE & P02469 & Sec. & Laminin beta-1 chain precursor (Laminin B1 chain). & 196.8 \\
\hline Hep. & LMA4_MOUSE & P97927; O88785 & Sec. & Laminin alpha-4 chain precursor. & 201.7 \\
\hline Hep. & PGG2_RAT & Q00657 & Sec. & Chondroitin sulfate proteoglycan NG2 precursor & 251.8 \\
\hline
\end{tabular}


Table 1 (continued)

\begin{tabular}{llllll}
\hline Fraction & SW.-Nr. & Access. Nr. & Status & Full Protein Name & kDa \\
\hline Sup. & FINC_RAT & P04937 & Sec. & Fibronectin precursor (FN). & 272.3 \\
Hep. & PGBM_MOUSE & Q05793 & Sec. & Basement memb.-spec. heparan sulfate proteoglycan & 398.0 \\
Hep.; Sup. & LMA5_MOUSE & Q61001 & Sec. & Laminin alpha-5 chain precursor. & 403.7 \\
Hep.; Sup. & LRP2_RAT & P98158 & Sec. & Low-density lipoprotein receptor-related protein 2 & 518.9 \\
\hline
\end{tabular}

concentration differed markedly from one subject to another and is constantly changing. This presents an everfluctuating glycemic stimulus that, when combined with intrinsic differences in $\beta$-cell function from one individual to another, leads to large variations in the plasma insulin profile between subjects as shown in [45]. Because the two primary variables of interest - plasma glucose and plasma insulin concentration - are changing simultaneously, it is difficult to draw any conclusion about insulin secretion or insulin sensitivity. Lastly, it is difficult to quantitate changes in hepatic glucose production after glucose ingestion because the rate of entry of glucose into circulation is unknown. In order to investigate the influence of the plasma glucose concentration on the PPY level, an OGTT with four healthy subjects were performed and all have revealed very similar results. Our investigations indicate that early insulin secretion, absolute insulin concentration at $30 \mathrm{~min}$, the incremental insulin concentration $(\Delta \mathrm{I})$ at $30 \mathrm{~min}$, or the incremental insulin concentration factored by the incremental glucose concentration $(\Delta \mathrm{I} / \Delta \mathrm{G})$ at $30 \mathrm{~min}$ had less predictive value for the $\beta$-cell function than the determination of PPY. PPY absolute concentration did not vary so much between the healthy individuals and it was shown that the secretion rate to be independent of the plasma glucose and insulin concentration. Furthermore, the PPY plasma concentration between the healthy individuals was very constant and only significantly elevated in T2D

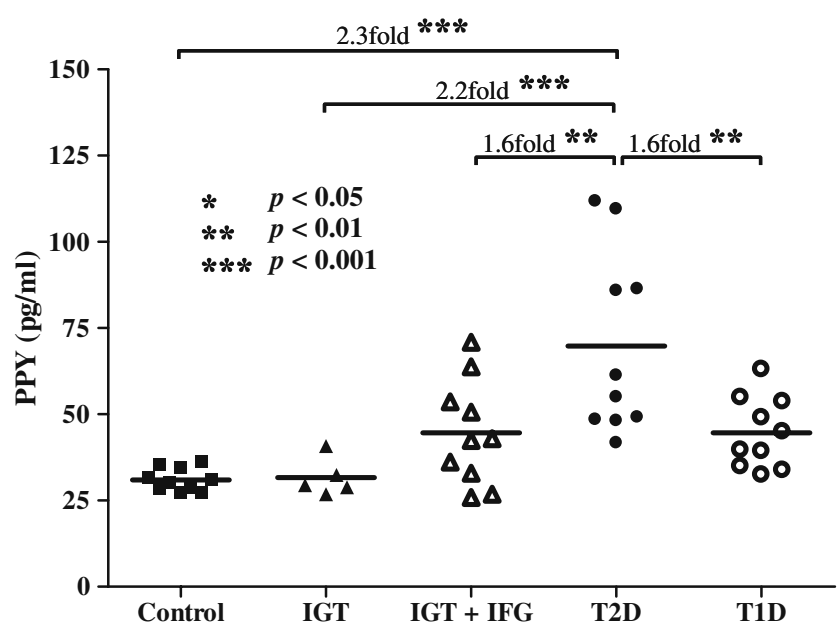

Fig. 7 Primary results: Validation of PPY in human blood / plasma samples in five different healthy donors by ELISA [43] and IGT + IFG individuals as shown in Fig. 7 and proved to be a better predictor of progression to T2D than OGTT. Since the plasma glucose concentration is the primary determinant of insulin secretion, it is obvious that fasting insulin and glucose concentration alone can not be used to predict the progression of normal-glucose-tolerant subjects to T2D. It is anticipated that our results and methods are able to establish a framework for the identification of additional marker candidates that need to be examined and provide a sound scientific basis for the identification and more precise definition of predictive biomarkers in T2D.

\section{Summary}

Individuals with T2D are characterized by abnormalities in insulin action and insulin secretion. However, despite intensive investigation, the proteins/peptides or genes responsible for the insulin resistance and impaired insulin secretion remain undefined. The candidate-proteins/genes approach has failed to identify any specific proteins or genes or combination of proteins/genes that can account for even a minority of adult cases of T2D. Although a number of laboratories have initiated genome- and proteome-wide

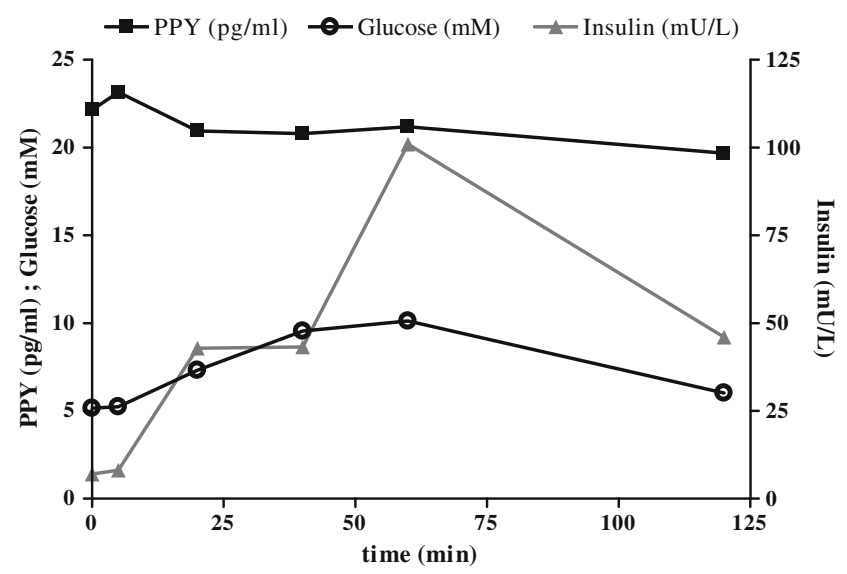

Fig. 8 Means of plasma glucose, insulin and PPY concentration during a $100 \mathrm{~g}$ OGTT in 4 healthy normal weight individuals. The results indicate that early insulin secretion has a less predictive value for the $\beta$-cell function than the determination of PPY levels. The absolute PPY concentration varies much less between healthy individuals and the results show that the secretion rate of PPY from the endocrine pancreas is independent of the plasma glucose and insulin concentration [43] 
search to identify potential susceptibility targets for T2D, consistent and reproducible linkage to predictive markers has yet to merge. It has been suggested that a more precise definition of the diabetic phenotype may prove useful in delineating diabetogenetic genes or gene products. This review should provide an inside into new analytical strategy for the specific enrichment and identification of secreted and pancreas specific proteins/peptides, which are responsible for abnormalities of insulin secretion in T2D and which may serve as predictive markers for $\beta$-cell function.

All together the analysis in [43] resulted in the identification of 391 different gene products derived from the INS cells. One hundred eighty-eight proteins bound to the heparin matrix, 101 of which were identified as secreted proteins with signaling functions and are listed in Table 1. Forty-four of the enriched proteins had not been detected by 2-DE without previous enrichment by combination of subcellular prefractionation and heparin chromatography. Heparin chromatography specifically enriched several enzymes that had not been identified before [46-49], like peptidyl-glycine alphaamidating monooxygenase, sodium/hydrogen exchanger 5, etc. The identified proteins can be divided into five classes:

- Hormones and related molecules (amylin, pancreatic polypeptide, neuroendocrine convertase)

- Protease inhibitors (e.g. cystatin)

- Secretory vesicle proteins (e.g. chromogranin, secretogranin)

- Cell adhesion or extracellular matrix proteins (e.g. protocadherin)

- Secreted enzymes (e.g. phospholipase)

From all identified secreted proteins only pancreatic polypeptide Y (PPY) was identified as pancreas specific, fulfilling the criteria of a predictive marker and hence was selected for further validation [43].

PPY (pancreatic polypeptide Y), a 78-amino acid peptide is synthesized in pancreatic islets of Langerhans and acts as a regulator of pancreatic and gastrointestinal functions. The peptide is structurally similar to another pancreatic hormone family consisting of neuropeptide Y (NPY) b, and peptide YY (PYY) which are closely related to each other. The propeptide is enzymatically cleaved to yield the mature active peptide with amidated $\mathrm{C}$-terminal ends while receptor binding and activation functions reside in the $\mathrm{N}$ - and $\mathrm{C}$-termini respectively. PPY occurs in neurons, intestinal endocrine cells, and the pancreas and exists as monomer and dimer. The physiological role for the icosapeptide has not yet been elucidated. PPY was identified as specifically secreted by the endocrine pancreas and it is mainly produced by $\beta$-and f-cells [47]. Its plasma concentration has been used as a marker of parasympathetic activity. Recent work in rodents suggests that there is both sympathetic and parasympathetic innervation of white adipose tissue and that parasympathetic activity is anabolic resulting in lipid accumulation [48]. It was examined whether in humans increased PP levels are associated with increased $\beta$-cell dysfunctions, and thereby early $\beta$-cell failure in T2D. Together with amylin, the peptide has been shown to be the precursor for the amyloid deposits frequently observed in patients with T2D [50-53]. At very high doses PPY inhibits insulin secretion by the perfused rat pancreas in vitro [54] and pancreatic PPY and amylin deposits have been shown to precede the appearance of glucose intolerance in spontaneously diabetic monkeys [55]. In the course of the study published in [43], the authors were able to demonstrate that elevated plasma PPY levels can be observed in IGT and IFG subjects, in glucose-intolerance first degree relatives of type 2 diabetic patients (see Fig. 7), and in animal models of diabetes. Although pancreatic and plasma PPY levels were significantly elevated, hyperglycemia and hyperinsulinemia were not observed (see Fig. 8). In summary, based on the findings/ observation, the evidence that PPY is responsible for some of the defects in pancreatic islets is very strong and it seems that PPY plays an important role in impaired $\beta$-cell function in $\mathrm{T} 2 \mathrm{D}$.

\section{References}

1. Garvey WT, Olefsky JM, Griffin J, et al. The effect of insulin treatment on insulin secretion and insulin action in type II diabetes mellitus. Diabetes. 1985;34:222-34.

2. Little RR, Rohlfing CL, Wiedmeyer HM, et al. The national glycohemoglobin standardization program: a five-year progress report. Clin Chem. 2001;47:1985-92.

3. Landin-Olsson M, Nilsson KO, Lernmark A, et al. Islet cell antibodies and fasting $\mathrm{C}$-peptide predict insulin requirement at diagnosis of diabetes mellitus. Diabetologia. 1990;33:561-8.

4. Meier C, Ladewig A, Keller U, et al. Clinical value of C-peptide determination. Praxis. 1997;86:1289-95.

5. Sacks DB, Bruns DE, Goldstein DE, et al. Guidelines and recommendations for laboratory analysis in the diagnosis and management of diabetes mellitus. Clin Chem. 2002;48:436-72.

6. Koehn J, Fountoulakis M, Krapfenbauer K. Multiple drug resistance associated with function of $\mathrm{ABC}$-transporters in diabetes mellitus: molecular mechanism and clinical relevance. Infect Disord Drug Targets. 2008;8:109-18.

7. Sattar N, Wannamethee SG, Forouhi NG. Novel biochemical risk factors for type 2 diabetes: pathogenic insights or prediction possibilities? Diabetologia. 2008;51:926-40.

8. Wannamethee SG, Shaper AG, Lennon L, et al. Hepatic enzymes, the metabolic syndrome, and the risk of type 2 diabetes in older men. Diabetes Care. 2005;28:2913-8.

9. Ding EL, Song Y, Malik VS, et al. Sex differences of endogenous sex hormones and risk of type 2 diabetes: a systematic review and meta-analysis. Jama. 2006;295:1288-99.

10. Smirnakis KV, Plati A, Wolf $M$, et al. Predicting gestational diabetes: choosing the optimal early serum marker. Am J Obstet Gynecol. 2007;196(410):e411-6. discussion 410 e416-7.

11. Pradhan AD, Manson JE, Rifai N, et al. C-reactive protein, interleukin 6 , and risk of developing type 2 diabetes mellitus. Jama. 2001;286:327-34. 
12. Kaye TB, Guay AT, Simonson DC. Non-insulin-dependent diabetes mellitus and elevated serum ferritin level. J Diabetes Complications. 1993;7:246-9.

13. Eshed I, Elis A, Lishner M. Plasma ferritin and type 2 diabetes mellitus: a critical review. Endocr Res. 2001;27:91-7.

14. Spranger J, Kroke A, Mohlig M, et al. Adiponectin and protection against type 2 diabetes mellitus. Lancet. 2003;361:226-8.

15. Vasseur F, Lepretre F, Lacquemant C, et al. The genetics of adiponectin. Curr Diab Rep. 2003;3:151-8.

16. Yamauchi $\mathrm{T}$, Kamon J, Ito $\mathrm{Y}$, et al. Cloning of adiponectin receptors that mediate antidiabetic metabolic effects. Nature. 2003;423:762-9.

17. Schmidt MI, Duncan BB, Vigo A, et al. Leptin and incident type 2 diabetes: risk or protection? Diabetologia. 2006;49:2086-96.

18. Festa A, D'Agostino Jr R, Tracy RP, et al. Elevated levels of acute-phase proteins and plasminogen activator inhibitor-1 predict the development of type 2 diabetes: the insulin resistance atherosclerosis study. Diabetes. 2002;51:1131-7.

19. Eliasson MC, Jansson JH, Lindahl B, et al. High levels of tissue plasminogen activator (tPA) antigen precede the development of type 2 diabetes in a longitudinal population study. The Northern Sweden MONICA study. Cardiovasc Diabetol. 2003;2:19.

20. Wannamethee SG, Sattar N, Rumley A, et al. Tissue plasminogen activator, von Willebrand factor, and risk of type 2 diabetes in older men. Diabetes Care. 2008;31:995-1000.

21. Meigs JB, O'Donnell CJ, Tofler GH, et al. Hemostatic markers of endothelial dysfunction and risk of incident type 2 diabetes: the Framingham Offspring Study. Diabetes. 2006;55:530-7.

22. Jacqueminet $\mathrm{S}$, Briaud I, Rouault $\mathrm{C}$, et al. Inhibition of insulin gene expression by long-term exposure of pancreatic beta cells to palmitate is dependent on the presence of a stimulatory glucose concentration. Metabolism. 2000;49:532-6.

23. Donath MY, Gross DJ, Cerasi E, Kaiser N. Hyperglycemia-induced beta-cell apoptosis in pancreatic islets of Psammomys obesus during development of diabetes. Diabetes. 1999;48:738-44.

24. Shafrir E, Ziv E, Mosthaf L. Nutritionally induced insulin resistance and receptor defect leading to beta-cell failure in animal models. Ann N Y Acad Sci. 1999;892:223-46.

25. Anello M, Rabuazzo AM, Degano C, et al. Fast reversibility of glucose-induced desensitization in rat pancreatic islets. Evidence for an involvement of ionic fluxes. Diabetes. 1996;45:502-6.

26. Marshak S, Leibowitz G, Bertuzzi F, et al. Impaired beta-cell functions induced by chronic exposure of cultured human pancreatic islets to high glucose. Diabetes. 1999;48:1230-6.

27. Busch AK, Cordery D, Denyer GS, et al. Expression profiling of palmitate-and oleate-regulated genes provides novel insights into the effects of chronic lipid exposure on pancreatic beta-cell function. Diabetes. 2002;51:977-87.

28. Carlsson C, Borg LA, Welsh N. Sodium palmitate induces partial mitochondrial uncoupling and reactive oxygen species in rat pancreatic islets in vitro. Endocrinology. 1999;140:3422-8.

29. Shimabukuro M, Zhou YT, Levi M, et al. Fatty acid-induced beta cell apoptosis: a link between obesity and diabetes. Proc Natl Acad Sci U S A. 1998;95:2498-502.

30. Mandrup-Poulsen T. beta-cell apoptosis: stimuli and signaling. Diabetes. 2001;50 Suppl 1:S58-63.

31. Asfari M, Janjic D, Meda P, et al. Establishment of 2mercaptoethanol-dependent differentiated insulin-secreting cell lines. Endocrinology. 1992;130:167-78.

32. Miyazaki J, Araki K, Yamato E, et al. Establishment of a pancreatic beta cell line that retains glucose-inducible insulin secretion: special reference to expression of glucose transporter isoforms. Endocrinology. 1990;127:126-32.

33. Praz GA, Halban PA, Wollheim CB, et al. Regulation of immunoreactive-insulin release from a rat cell line (RINm5F). Biochem J. 1983;210:345-52.
34. Karlsson K, Cairns N, Lubec G, et al. Enrichment of human brain proteins by heparin chromatography. Electrophoresis. 1999;20:2970-6.

35. Shin JH, Krapfenbauer K, Lubec G. Large-scale identification of cytosolic mouse brain proteins by chromatographic prefractionation. Electrophoresis. 2006;27:2799-813.

36. Conrad EH. Heparin-binding proteins. San Diego: Academic Press, Inc.; 1998.

37. Pollak D, Krapfenbauer K, Fountoulakis M, et al. Expressional pattern of known and predicted signaling proteins in seven human cell lines. J Chromatogr. 2004;808:185-208.

38. Shin JH, Krapfenbauer K, Lubec G. Column chromatographic prefractionation leads to the detection of 543 different gene products in human fetal brain. Electrophoresis. 2005;26:2759-78.

39. Cagney G, Park S, Chung C, et al. Human tissue profiling with multidimensional protein identification technology. J Proteome Res. 2005;4:1757-67.

40. Florens L, Washburn MP. Proteomic analysis by multidimensional protein identification technology. Methods in molecular biology Clifton, NJ, 328, 159-175. 2006.

41. Fountoulakis M, Juranville JF, Jiang L, et al. Depletion of the high-abundance plasma proteins. Amino acids. 2004;27:249-59.

42. Lubec G, Krapfenbauer K, Fountoulakis M. Proteomics in brain research: potentials and limitations. Prog Neurobiol. 2003;69:193-211.

43. Koehn J, Turhani D, Krapfenbauer K. Potential predictive biomarkers for the dysfunction of beta-cells in type 2 diabetes In: Golubnitschaja $\mathrm{O}$ (ed) Predictive Diagnostics and personalized Treatment, Dream or Reality? Nova Publisher. 2009.

44. Fountoulakis M, Langen H. Identification of proteins by matrixassisted laser desorption ionization-mass spectrometry following in-gel digestion in low-salt, nonvolatile buffer and simplified peptide recovery. Anal Biochem. 1997;250:153-6.

45. Peyrl A, Krapfenbauer K, Slave I, et al. Protein profiles of medulloblastoma cell lines DAOY and D283: identification of tumor-related proteins and principles. Proteomics. 2003;3:1781-800.

46. Langen $\mathrm{H}$, Roder $\mathrm{D}$, Juranville $\mathrm{JF}$, et al. Effect of protein application mode and acrylamide concentration on the resolution of protein spots separated by two-dimensional gel electrophoresis. Electrophoresis. 1997;18:2085-90.

47. Wang F, Permet J, Ostenson CG. Islet amyloid polypeptide regulates multible steps in stimulus-secretion coupling of beta cells in rat pancreatic islets. Pancreas. 2000;20(3):264-9.

48. Karlsson E, Stridsberg M, Sandler S. Leptin regulation of islet amyloid polypeptide secretion from mouse pancreatic islets. Biochem Pharmcol. 1998;15(56(10)):1339-46.

49. Golubnitschaja O, Flammer J. What are the biomarkers for glaucoma? Surv Ophthalmol. 2007;52 Suppl 2:S155-61.

50. Golubnitschaja O, Yeghiazaryan K, Wunderlich K, et al. Disease proteomics reveals altered basic gene expression regulation in leukocytes of Normal-Tension and Primary Open-Angle glaucoma patients. Proteomics Clin Appl. 2007;1:1316-23.

51. Clark A. Islet amyloid and type 2 diabetes. Diabet Med. 1989;6:561-7.

52. Nishi M, Sanke T, Nagamatsu S, et al. Islet amyloid polypeptide. A new beta cell secretory product related to islet amyloid deposits. J Biol Chem. 1990;265:4173-6.

53. Westermark P, Wernstedt C, Wilander E, et al. Amyloid fibrils in human insulinoma and islets of Langerhans of the diabetic cat are derived from a neuropeptide-like protein also present in normal islet cells. Proc Natl Acad Sci U S A. 1987;84:3881-5.

54. Ohsawa H, Kanatsuka A, Yamaguchi T, et al. Islet amyloid polypeptide inhibits glucose-stimulated insulin secretion from isolated rat pancreatic islets. Biochem Biophys Res Commun. 1989;160:961-7.

55. Howard Jr CF. Longitudinal studies on the development of diabetes in individual Macaca nigra. Diabetologia. 1986;29:301-6. 